\title{
The Status Quo and Prospect of Model-free Adaptive Control
}

\author{
Hu Rong \\ School of mechanical and electrical engineering, Guangdong Engineering Polytechnic, Guangdong, China, \\ 510520
}

Keywords: No model; adaptive control; current situation

\begin{abstract}
In order to solve the problem that the general control engineering course lags behind the information age and the actual needs of engineering, this paper explores new ways and new methods of the closed-loop control experiment of brushless DC motor speed. This paper will discuss the model-free adaptive control method and STM32 single-chip microcomputer. Brushed DC motor speed closed-loop adaptive control method aims to expand students' knowledge, strengthen the cognition of hotspot control methods, connect theory with practice, and stimulate students' innovative thinking on the application of control methods.
\end{abstract}

\section{Introduction}

The spirit of innovation and the cultivation of creative practice ability are the key to education. The traditional single control engineering experiment is difficult to meet the requirements of the high-speed development society for talent training. Due to the high cost of the experimental engineering course supporting hardware equipment, the special machine is dedicated, the flexibility of the experimental project formation is low, and most of the experimental projects have to be developed around MATLAB simulation. The control engineering course experiment of MATLAB simulation alone lacks the necessary hardware equipment, which makes students unable to understand the connection between the course and the actual engineering problems, which is extremely unfavorable for cultivating their hands-on ability and application ability for engineering practice. Therefore, it is necessary to reform experimental methods, research and design an experimental device that is cost-effective, can be controlled by MATLAB, and can stimulate students' innovative thinking ${ }^{[1]}$.

The experimental content of the general control engineering course is attached to the curriculum establishment. There are too many simulation contents, the experimental methods and object settings are relatively traditional, the experimental equipment functions are relatively simple, lack of theoretical connection and reality, lack of modularity and intelligence, and equipment resources cannot be shared. Wait, lag behind the information age and the actual needs of the project. The brushless DC motor combines many advantages of traditional DC motor and AC motor, and has the advantages of high reliability, high efficiency, long life, good torque and torque characteristics, good regenerative braking and simple maintenance. It has been used in defense industry, automobile and electric bicycle. Industries such as home appliances, industrial process control, precision machine tools, robotics, aircraft models and environmental protection machinery are widely used. The model-free adaptive control method is to realize the adaptive control system by calculating the input and output data of the controlled system and generating a reasonable system driving amount, so that the deviation between the actual value and the set value of the system output becomes smaller. The method of control ${ }^{[2]}$. The controller based on model-free adaptive control has the advantages of good tracking performance and easy implementation. It has been widely used in the chemical industry, machinery manufacturing, aerospace and industrial robots, and has the development trend of replacing PID controller.

\section{Status of model-free adaptive control}

In recent years, dynamic system control with a wide range of parameter uncertainties is receiving 
more and more attention. Due to factors such as large-scale uncertainty of system parameters, the system changes are complicated. In this context, more the multi-model adaptive control of the model to cover the dynamic characteristics of the system has been developed. Based on the selected system performance indicators, the most suitable controller is used to control the system in real time. The idea of multi-model was first proposed by Magill in the 1960s and applied to state estimation of stochastic systems with uncertain parameters, namely multiple model adaptive estimation (MMAE). In the 1970s by Lainiotis et al. And Athans et al. proposed multiple model adaptive control (MMAC), which is a multi-model control algorithm based on posterior probability weighting. Then, Badr et al. designed each controller with quadratic optimal index to form a weighted multi-model controller. It was applied in the control of the aircraft and the adjustment of hemodynamic variables. Chaudhuri et al used this method to control the power. Damping of low-frequency oscillations in the system. Later, Narendra et al. proposed switching multi-model adaptive control. The basic idea is to construct multiple fixed models offline to cover the uncertain space of the controlled object, and an adaptive model to improve the dynamic performance of the system. With the development of controller design such as predictive control, robust adaptive control and fuzzy PID control, the control strategy combined with the weighted multi-model method improves the control performance of the system ${ }^{3]}$. Fuzzy pid control as shown in Equation 1

$$
u(t)=K_{p}\left[e(t)+\frac{1}{T i} \int e(t) d t+T d \frac{d e(t)}{d t}\right]
$$

In recent years, the literature proposes a multi-model adaptive hybrid control that can achieve a smooth transition between controllers. The weighted values of the outputs of multiple controllers are generated based on the parameter estimates of the controlled objects. The literature extends the above results to discrete time. System. Cao Xufeng et al. combined the advantages of switching and hybrid control to further improve the transient performance of the system. In addition, in order to reduce the number of model sets and make full use of the system information provided by the model, Han and Narendra proposed a multi-model two-stage adaptive method (multiple Models with second level adaptation, MMSLA), which actually introduces weighting ideas at the level of parameter estimation. At the same time, the method is applied in many fields such as high-speed trains and chemical processes. Compared with multi-model adaptive control based on handover, weighted multiple model adaptive control (WMMAC) can be regarded as a multi-model control system based on "soft handover". In fact, for random control systems with uncertain parameters, this method can be considered. However, because of the weighted form of the controller, it is still very difficult to prove the stability of the weighted multi-model adaptive control system despite decades of theoretical and experimental research. In recent years, similar literatures and other related literatures have given the stability and convergence proof of weighted multi-model adaptive control systems based on virtual equivalent system theory. However, the weighted multi-model adaptive control is only for the model set. The fixed model constitutes and contains the real object model, and its corresponding stability analysis is also based on this situation. When the model set does not contain the real object model, the control system is difficult to meet the control requirements ${ }^{[4]}$. The application trend of the control method changes as shown in Figure 1.

In order to solve the above problems, based on the literature, a weighted multi-model adaptive control system with self-correction model is constructed, and the convergence proof of the weighted algorithm is given. After that, based on the fixed model and self control system of the model set consisting of the correction model proves the stability and convergence of the control system based on the virtual equivalent system theory when the parameters of the controlled object are unknown linear time constant and parameter jump, and the literature weights are relaxed. In the stability analysis of the model adaptive control system, the model set is only the constraint of the fixed model, and the theoretical application range of the guaranteed weighted multi-model adaptive control system is expanded ${ }^{[5]}$. 


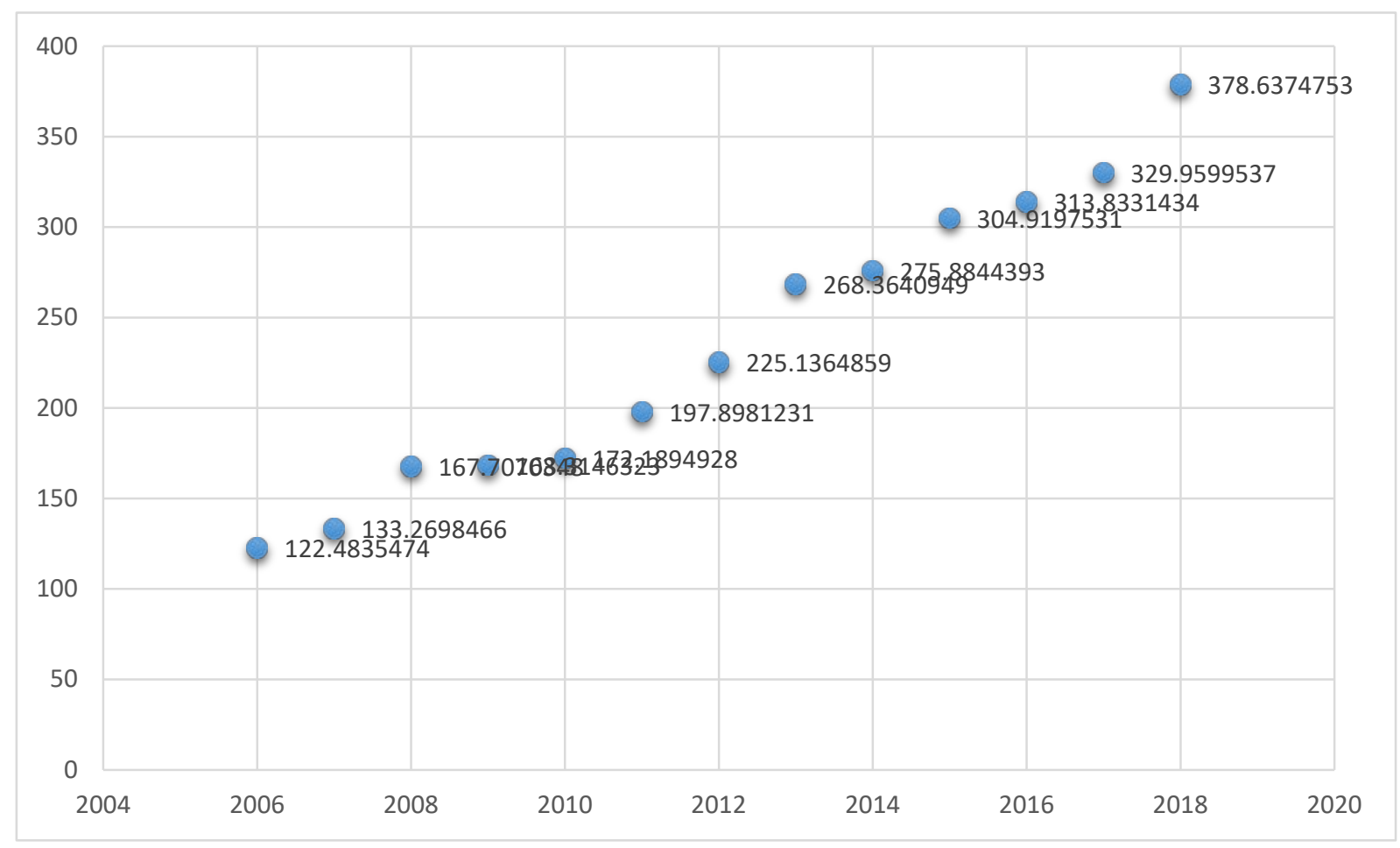

Figure 1: Application trend of the control method changes

\section{Adaptive control method}

In practical applications, the controller based on the model-free adaptive control method is actually a nonlinear dynamic negative feedback control link or module. In this paper, based on the set value of the speed and the measured value of the measured speed of the speed measuring device, the model-free adaptive controller is used to calculate the duty cycle of the PWM (Pulse Width Modulation) signal, and the PWM based on the duty cycle is used. The signal is applied to the brushless motor power drive module to complete the closed-loop speed control, forming a closed-loop control system for the brushless DC motor speed. The brushless DC motor speed closed-loop control system can be reduced to a single-input single-output discrete-time nonlinear system. Since the pseudo partial derivative $\varphi(\mathrm{k})$ is unknown, in the actual control system, the online estimate of $\varphi(\mathrm{k})$ is often used instead. The method of finding online estimation values in the model-free adaptive control law is generally called a pseudo partial derivative identification algorithm. For the controlled system studied in this paper, the goal of the pseudo partial derivative identification algorithm is to punish the variation of the system pseudo partial derivative on the basis of making the infinite approximation $y(k)-y(k-1)$, that is, the value cannot be Too big. Where $\rho$ is an adjustable step sequence. Therefore, the closed-loop control method of the brushless DC motor speed based on the model-free adaptive controller does not need to construct the mechanism model and the process state equation, nor does it need complicated parameter tuning and fuzzy rule tables, only the input of the controlled system is required. By outputting data, the adaptive control of the controlled system can be completed, which has the advantages of not relying on a specific model, being easy to implement, online adaptive, smooth process control, and good control effect. The implementation and application of the model-free adaptive control method in the control engineering course experiment, bringing students from the PID control experiment into the model-free adaptive control experiment, will greatly stimulate students' innovative thinking and train students to control the controlled object. Diversified control methods and foundations for engineering practical applications.

Resources such as SPI, USB, Flash, and TIM enable communication, acquisition, and drive of the entire control system. The main functions of the system include: using the superior computing power of the processor to realize the model-free adaptive controller; using the high-address area of 
the processor to implement the system parameter memory module to save the control algorithm parameters; using the ADC to feedback back to the rotation speed detecting device The speed voltage analog quantity is sampled, the digital voltage is converted by the speed calculation module to obtain the measured speed; the SPI communication interface is used to realize the speed setting, and the setting of the desired output of the system in the modeless adaptive controller is completed; using the high speed USB2.0 The controller stores the adaptive control parameters into the parameter memory module, and transmits the real-time rotational speed and duty cycle back to the upper computer to complete the system tuning and parameter setting; using the universal TIM timer and the duty ratio of the model-free adaptive controller output, Modulation and generation of PWM signals; using a three-phase bridge drive circuit and a power amplifier circuit to achieve power amplification of the PWM signal to drive the motor to rotate.

\section{Conclusion}

The brushless DC motor speed closed-loop controller based on the model-free adaptive control method has strong adaptive ability, can dynamically adjust the controller weight parameter according to the input and output changes during the control process, and can complete the speed closed-loop control well. It has strong practicability and has important reference significance for similar research and application. Based on the model-free adaptive control method, the closed-loop control of the brushless DC motor speed in the control engineering course experiment solves the problem that the general control engineering course experiment lags behind the information age and the actual needs of the project, and deepens the content of the control engineering experiment course. Expanded students' innovative thinking.

\section{References}

[1] Irja Marije de Jong,Frank Kupper,Jacqueline Broerse. Unscripted Responsible Research and Innovation: Adaptive space creation by an emerging RRI practice concerning juvenile justice interventions[J]. Life Sciences, Society and Policy,2018,14(1).

[2] Hao Li,Yidu Yang. Adaptive Morley element algorithms for the biharmonic eigenvalue problem[J]. Journal of Inequalities and Applications,2018,2018(1).

[3] Peipei Zhou,Shuiming Cai. Adaptive exponential lag synchronization for neural networks with mixed delays via intermittent control[J]. Advances in Difference Equations,2018,2018(1).

[4] Khaled Obaid Al Ali,Nemanja Ilić,Miloš S. Stanković,Srdjan S. Stanković. Consensus-based distributed adaptive target tracking in camera networks using Integrated Probabilistic Data Association[J]. EURASIP Journal on Advances in Signal Processing,2018,2018(1).

[5] Biwen Li,Nengjie Wang,Xiaoli Ruan,Qiujin Pan. Pinning and adaptive synchronization of fractional-order complex dynamical networks with and without time-varying delay[J]. Advances in Difference Equations,2018,2018(1). 